\title{
Le Programme de traitements antalgiques en urgence mis à jour et enrichi avec de nouvelles fiches
}

\section{The Analgesic Treatments Programme for Emergency Departments - updated and improved with new data sheets}

C) Springer-Verlag France 2012

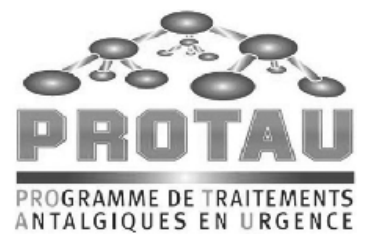

L'Institut UPSA de la douleur annonce la mise à jour du Programme de traitements antalgiques en urgence (PROTAU).

Spécialement conçu pour les urgentistes et les professionnels de santé confrontés à l'urgence, le programme PROTAU propose des procédures antalgiques spécifiques des principaux syndromes douloureux rencontrés en situation d'urgence en fonction de l'origine de la douleur.

\section{PROTAU : un succès auprès des professionnels de santé}

C'est devant le large succès de ce programme lancé en 2007 (plus de 12000 classeurs PROTAU distribués ou téléchargés en cinq ans) que les membres du Conseil scientifique de l'Institut UPSA de la douleur ont décidé la mise à jour et l'enrichissement de ce programme.

\section{Fiches pratiques par type de douleur}

L'ensemble de ces procédures se présente sous forme de fiches claires et pratiques, appuyées sur une bibliographie scientifique et validées par un groupe d'experts présidé par le Dr Agnès Ricard-Hibon (Smur, SAU-Beaujon, Clichy).
Ce groupe a mis à jour une vingtaine de protocoles rédigés en fonction de cas pratiques se présentant aux urgences, comme par exemple brûlures, coliques néphrétiques ou encore des douleurs abdominales chez l'adulte ou l'enfant. En outre, chaque fiche contient des spécificités soins infirmiers ainsi que des algorithmes détaillés de prise en charge de la douleur pour faciliter le travail au quotidien des urgentistes.

\section{De nouvelles fiches pratiques à disposition}

Trois nouvelles fiches ont également été créées afin d'enrichir le contenu du programme PROTAU. La première traite de l'utilisation du mélange équimolaire de protoxyde d'azote et d'oxygène (MEOPA), la deuxième est consacrée à l'anesthésie locale topique (EMLA) et la troisième est dédiée à la place de l'échographie dans l'anesthésie locale régionale (ALR).

À noter que l'ensemble des fiches de ce programme est disponible sur le site Internet de l'Institut UPSA de la douleur : www.institut-upsa-douleur tant en téléchargement en format PDF qu'en liens QR codes pour une lecture sous forme de e-books directement sur smartphone. 